\title{
Bloqueio Extraconal para Facectomia com Implante de Lente Intra-Ocular: Influência do Fentanil Associado ao Anestésico Local na Qualidade do Bloqueio e na Analgesia Pós-Operatória *
}

\section{Extraconal Block for Cataract Extraction Surgery with Implantation of Intraocular Lens: Contribution of Fentanyl Associated to Local Anesthe- tics for Quality of Block and Postoperative Analgesia}

\author{
Daniel Espada Lahoz ${ }^{1}$; Eloisa Bonetti Espada ${ }^{2}$; José Carlos Almeida Carvalho, TSA ${ }^{3}$
}

\section{RESUMO}

Lahoz DE, Espada EB, Carvalho JCA - Bloqueio Extraconal para Facectomia com Implante de Lente Intra-Ocular: Influência do Fentanil Associado ao Anestésico Local na Qualidade do Bloqueio e na Analgesia Pós-Operatória

JUSTIFICATIVA E OBJETIVOS: A anestesia locorregional para cirurgias oftalmológicas oferece vantagens, como: mínimas alterações fisiológicas, anestesia completa, bloqueio dos reflexos oculares, pequena incidência de náuseas e vômitos, menor tempo de recuperação e analgesia pós-operatória. A preocupação constante com a qualidade do bloqueio, assim como da abordagem da analgesia pós-operatória deve ficar sob a responsabilidade do anestesiologista. O objetivo deste estudo foi avaliar se o fentanil contribui na qualidade do bloqueio extraconal e na analgesia pós-operatória de facectomias com implante de lente intra-ocular.

MÉTODO: Estudou-se a associação do fentanil e bupivacaína a $0,75 \%$ na qualidade do bloqueio ocular e na analgesia pós-operatória em 164 pacientes submetidos a facectomia com implante de lente intra-ocular (técnica extracapsular), de ambos os sexos com homogeneidade de parâmetros antropométricos, olho operado, classificação do estado físico (ASA) e índice de risco cardíaco de Goldman. Os pacientes foram distribuídos em dois grupos (82 pacientes em cada grupo) por sorteio de forma aleatória, com e sem fentanil. Avaliou-se a qualidade do bloqueio por: aparecimento da dor no per-operatório, manutenção de movimentação das pálpebras ou do globo ocular, persistência do reflexo de Bell, número de bloqueios realizados para a obtenção de condições cirúrgicas e avaliação do bloqueio pelo cirurgião. A analgesia pós-operatória foi avaliada pela necessidade de complementação analgésica pelo paciente.

\footnotetext{
* Recebido do (Received from) Hospital Santa Cruz, Hospital Universitário da Faculdade de Medicina da Universidade de São Paulo (FMUSP), São Paulo, SP

1. Mestre em Medicina pela FMUSP; Coordenador do Serviço de Anestesia em Oftalmologia da UNIFESP; Assistente da Disciplina de Anestesio logia do HC da FMUSP;Anestesiologia do Serviço de Oxigênioterapia: Anestesiologia e Inaloterapia (SOANIL) do Hospital Santa Cruz

2. Doutora em Medicina pela FMUSP; Diretora do Serviço de Anestesiologia do Hospital Universitário da USP

3. Livre Docente pela Disciplina de Anestesiologia da FMUSP
}

Apresentado (Submitted) em 05 de setembro de 2002

Aceito (Accepted) para publicação em 08 de janeiro de 2003

Endereço para correspondência (Correspondence to)

Dr. Daniel Espada Lahoz

R. Dr. Nicolau de Souza Queiroz, 297/121 Aclimação

04105-002 São Paulo, SP

E-mail: espada@ajato.com.br

(c) Sociedade Brasileira de Anestesiologia, 2003
RESULTADOS: Fentanil associado à solução anestésica no bloqueio extraconal aumentou significativamente o bloqueio do músculo reto medial (com fentanil - 17,1\%, sem fentanil$32,9 \%$ e diminuiu o consumo de analgésicos no período pós-operatório (uso de analgésicos com fentanil - 20,7\%, não uso de analgésicos com fentanil - 41,5\%).

CONCLUSÕES: Nas condições deste estudo o fentanil melhorou a qualidade do bloqueio quanto à motilidade do músculo reto medial e diminuiu a necessidade de analgésicos no pós-operatório.

Unitermos: ANALGÉSICOS, Opióides: fentanil; ANESTÉSICOS, Local: bupivacaína; CIRURGIA, Oftalmológica: catarata; TÉCNICAS ANESTÉSICAS, Regional: bloqueio extraconal

\section{SUMMARY}

Lahoz DE, Espada EB, Carvalho JCA - Extraconal Block for Cataract Extraction Surgery with Implantation of Intraocular Lens: Contribution of Fentanyl Associated to Local Anesthetics for Quality of Block and Postoperative Analgesia

BACKGROUND AND OBJECTIVES: Loco-regional anesthesia for cataract extraction surgery offers as advantages minimum physiological changes, complete anesthesia, eye reflexes blockade, lower incidence of nausea and vomiting and shorter recovery time, in addition to postoperative analgesia. Continuous concern with blockade quality as well as with postoperative analgesia is responsibility of the anesthesiologist. This study aimed at evaluating whether fentanyl has contributed to blockade quality and postoperative analgesia in cataract extraction surgery with implantation of intraocular lens.

METHODS: The association of fentanyl and $0.75 \%$ bupivacaine for eye blockade and postoperative analgesia was evaluated in 164 patients undergoing cataract extraction with implantation of intraocular lens (extracapsular technique). Patients were homogeneous in gender, demographics, operated eye, ASA physical status and Goldman's cardiac risk index. Patients were randomly allocated in two groups (82 patients each): with or without fentanyl. Blockade quality was evaluated according to the following parameters: intraoperative pain; eyelid and/or eyeball movement; Bell's reflex persistence; number of blocks needed to produce akinesia and surgeons evaluation of blockade. Postoperative analgesia was evaluated by patients' request for additional postoperative analgesia.

RESULTS: Results have shown that fentanyl has significantly improved medial rectus muscle blockade quality (with fentanyl 17.1\%; without fentanyl - 32.9\%) and has decreased postoperative analgesics consumption (analgesics with fentanyl - 20.7\%; no analgesics with fentanyl - 41.5\%).

CONCLUSIONS: In the conditions of this study, fentanyl has improved block quality, has decreased medial rectus muscle motility and the need for postoperative analgesics.

Key Words: ANALGESICS, Opioids: fentanyl; ANESTHETICS, Local: bupivacaine; ANESTHETIC TECHNIQUES, Regional: extraconal block; SURGERY, Ophthalmologic: cataract extraction 


\section{INTRODUÇÃO}

O s pacientes quando submetidos a qualquer tipo de cirurgia, embora desejem anestesia no período per-operatório, muitas vezes toleram e acabam por considerar normal o sofrimento causado pela dor pós-operatória ${ }^{1}$. Nos últimos anos tem havido, por parte dos anestesiologistas, maior preferência por técnicas de anestesia regional. Esse maior interesse provavelmente decorre da simplicidade das técnicas, do maior conhecimento dos seus mecanismos de ação e, ainda, do aparecimento de novos fármacos que, utilizados tanto na anestesia como na analgesia pós-operatória, têm aumentado a segurança do ato anestésico-cirúrgico e o conforto do paciente no período pós-operatório ${ }^{2}$.

Embora não se disponha de evidências da existência de receptores opióides no bulbo ocular ${ }^{3,4}$, desconhece-se nos bloqueios para cirurgia oftalmológica se a utilização de opióides atuaria como em outras técnicas regionais. O objetivo deste estudo foi avaliar a contribuição do fentanil na qualidade do bloqueio oftalmológico extraconal e na analgesia pós-operatória.

\section{MÉTODO}

Após aprovação pelas Comissões de Ética em Pesquisa dos hospitais onde foi realizado o estudo, foram incluídos 164 pacientes de ambos os sexos, com idades entre 23 e 92 anos, estado físico ASA I a IV distribuídos aleatoriamente em dois grupos: Grupo CF, que recebeu bloqueio extraconal com bupivacaína a $0,75 \%$ associada a fentanil; grupo SF, que recebeu bupivacaína a $0,75 \%$ sem fentanil.

Todos os pacientes apresentavam indicação cirúrgica de facectomia extracapsular com implante de lente intra-ocular, sendo os bloqueios realizados pelo mesmo anestesiologista. A solução anestésica era preparada por uma auxiliar de enfermagem treinada para tal, a qual realizava o sorteio e preparava a solução, sendo então entregue ao anestesiologista rotulada por um número, que era confrontado com os demais dados do paciente após o término da coleta destes para se definir à qual grupo pertencia.

Todos os pacientes foram monitorizados com cardioscopia, pressão arterial não invasiva e oximetria de pulso, após o que realizou-se venóclise periférica em membro superior com cateter calibre 20 ou $22 \mathrm{G}$, sendo iniciada a hidratação pela infusão de solução de Ringer com lactato na velocidade aproximada de $3 \mathrm{ml} \cdot \mathrm{min}^{-1}$.

A solução anestésica utilizada foi bupivacaína a 0,75\% com hialuronidase (100 UTR. $\left.\mathrm{ml}^{-1}\right)$, no volume total de $7 \mathrm{ml}$. A hialuronidase foi diluída com a própria bupivacaína na proporção de 2.000 UTR para cada $20 \mathrm{ml}$ de bupivacaína a $0,75 \%$. No grupo com fentanil era acrescentada à solução anestésica $0,5 \mathrm{ml}$ de citrato de fentanil $(25 \mu \mathrm{g})$. No grupo sem fentanil, adicionava-se $0,5 \mathrm{ml}$ de água destilada. Para a realização do bloqueio foi utilizada agulha hipodérmica descartável calibre $7 \mathrm{com} 40 \mathrm{~mm}$ de comprimento.

Os pacientes eram sedados com $50 \mu \mathrm{g}$ de fentanil, por via venosa, e doses tituladas de propofol a $0,5 \%$ até a perda do re- flexo ciliar, quando era realizado o bloqueio extraconal. Quando desperto da sedação era submetido à avaliação quanto a acinesia e à analgesia.

A via de acesso extraconal superior teve como referência para punção 1 a $2 \mathrm{~mm}$ da borda lateral da chanfradura supra-orbitária, com o direcionamento da agulha para a órbita até que a mesma transfixasse a pele e o tecido celular subcutâneo. Reposicionou-se a ponta da agulha com direção à fissura orbitária superior, aproximando-a ao máximo das estruturas ósseas. Após a aproximação, que ocorreu entre 30 e 35 $\mathrm{mm}$, retrocedeu-se a agulha por aproximadamente 1 a $2 \mathrm{~mm}$, onde foi injetada a solução anestésica.

Avia de acesso extraconal inferior teve como local de punção a junção do terço lateral com o terço medial da borda orbitária inferior. Direcionou-se a ponta da agulha para a fissura orbitária inferior, sendo introduzida paralelamente ao assoalho da órbita por aproximadamente $30 \mathrm{~mm}$, onde se depositou a solução anestésica.

Na falha do bloqueio proposto associou-se bloqueio complementar. Ocorrendo falha do bloqueio extraconal superior complementava-se com o bloqueio extraconal inferior e vice-versa. A solução anestésica utilizada foi bupivacaína a $0,75 \%$ com hialuronidase (100 UTR. $\mathrm{ml}^{-1}$ ), no volume total de $3 \mathrm{ml}$. Na persistência da falha do bloqueio complementar procedia-se ao terceiro bloqueio, intraconal ou retrobulbar, com bupivacaína a $0,75 \%$ com hialuronidase (100 UTR.ml ${ }^{-1}$ ), no volume total de $3 \mathrm{ml}$.

Para o bloqueio intraconal utilizou-se como via de acesso a junção do terço lateral com o terço medial da borda orbitária inferior. Após a ponta da agulha ter ultrapassado o equador do bulbo ocular, esta foi reposicionada súpero-medialmente em direção à junção do terço medial com o terço lateral da borda orbitária superior do olho contralateral. Este procedimento ocorreu até que houvesse a sensação da perfuração de uma folha de papel. Neste local foi administrada a solução anestésica.

Nos casos em que a falha era apenas motora palpebral, complementava-se com bloqueio modificado de van Lint, utilizando-se apenas $3 \mathrm{ml}$ de bupivacaína a 0,75\%.

O bloqueio de van Lint modificado foi realizado injetando-se a solução anestésica aproximadamente $1 \mathrm{~cm}$ da borda lateral orbital no subcutâneo, diretamente sobre o periósteo, com direcionamento da agulha caudal e posteriormente cefálica com injeção de $1,5 \mathrm{ml}$ em cada direção.

Em seqüência à injeção do anestésico local, em qualquer fase, era realizada pressão digital firme sobre o bulbo ocular por aproximadamente 2 a 3 minutos, após a qual foi colocado um peso de $650 \mathrm{~g}$ no olho a ser operado com a finalidade de diminuir a pressão intraocular e facilitar a dispersão do anestésico local.

Com intenção de avaliar a qualidade do bloqueio foram estudados os seguintes parâmetros: dor per-operatória, manutenção da mobilidade das pálpebras, movimentação do globo (considerando-se os músculos extra-oculares associados e em separado), persistência do reflexo de Bell, número de bloqueios intracavitários complementares necessários para se obter condições cirúrgicas e a avaliação do bloqueio 
pelo cirurgião, o qual não estava ciente nem da solução injetada nem da via do bloqueio utilizado, de acordo com a seguinte escala:

1) Anestesia completa, paralisia ocular total, paciente colaborativo;

2) Anestesia completa, paralisia ocular incompleta, paciente colaborativo;

3) Anestesia incompleta, paralisia ocular total, paciente com desconforto cirúrgico;

4) Anestesia incompleta, paralisia ocular incompleta, paciente com desconforto.

Aanalgesia nas primeiras 72 horas do pós-operatório foi avaliada pela necessidade de complementação analgésica solicitada pelo próprio paciente, com administração de dipirona ou ácido acetil salicílico por via oral. Nos pacientes que necessitaram do uso dessa medicação complementar o tempo transcorrido do início do bloqueio até a ingestão do fármaco também foi avaliado.

Para a analise estatística utilizou-se o teste do Qui-quadrado com o objetivo de verificar diferenças na distribuição de um atributo categorizado em função de outro também categorizado, em alguns casos o teste não pôde ser aplicado em função da baixa freqüência observada em alguns atributos. Quando o total de casos ultrapassou 40, foi utilizado o teste Qui-quadrado com correção de Yates, assim como nos casos entre 20 e 40 , sendo que nenhum atributo apresentou freqüência menor que 5. Quando o número de casos era menor que 20 e a freqüência do atributo menor que 5, utilizou-se o teste de Fisher.

Para verificar se os grupos diferiam quanto a idade, peso, altura e tempo em minutos do início do bloqueio até o aparecimento de dor no período pós-operatório utilizou-se a Análise de Variância (ANOVA).

Em todos os testes estatísticos utilizou-se nível de significância de 5\%, sendo os testes realizados com auxílio do software estatístico SPSS para Windows versão 6.0.

\section{RESULTADOS}

Os 164 pacientes eram de ambos os sexos ( 78 do sexo masculino e 86 do sexo feminino), com limite de idade inferior de 23 anos e superior de 92 anos, apresentaram distribuição homogênea entre os grupos quanto aos dados antropométricos e olho operado (Tabela I).

Quanto ao estado físico e o índice de risco cardíaco de Goldman não houve diferença estatisticamente significante entre os grupos prevalecendo o estado físico ASAlle 1 no índice de risco cardíaco de Goldman (Tabela I).

Não se observou diferença estatística quanto à presença de dor no per-operatório entre os grupos nos diferentes momentos estudados (abertura da esclera, último ponto de sutura da esclera e injeção subconjuntival de gentamicina). Destaca-se a baixa incidência em todos os momentos sendo um pouco mais elevada por ocasião da injeção subconjuntival da gentamicina como pode ser visto na figura 1.
Tabela I - Dados Antropométricos, Olho Operado, Estado Físico e Índice de Risco Cardíaco de Goldman dos Pacientes

\begin{tabular}{lcc}
\hline Variáveis & $\begin{array}{c}\text { Grupo CF } \\
(\mathrm{n}=82)\end{array}$ & $\begin{array}{c}\text { Grupo SF } \\
(\mathrm{n}=82)\end{array}$ \\
\hline Idade (anos) & $69,7 \pm 10,3$ & $68,4 \pm 12,8$ \\
Peso $(\mathrm{kg})^{*}$ & $67,4 \pm 16,2$ & $65,9 \pm 12,6$ \\
Altura (cm)* & $164,2 \pm 11,3$ & $161,7 \pm 10,7$ \\
Sexo (\%) & 50 & \\
$\quad$ Masculino & 50 & 54,9 \\
$\quad$ Feminino & & 45,1 \\
Olho Operado (\%) & 54,9 & \\
$\quad$ Direito & 45,1 & 46,3 \\
$\quad$ Esquerdo & & 53,7 \\
Estado Físico (\%) & 18,3 & 32,9 \\
$\quad$ ASA I & 73,2 & 56,1 \\
ASA II & 6,1 & 11 \\
ASA III & 2,4 & 0 \\
ASA IV & & 89 \\
Goldman (\%) & 86,6 & 11 \\
1 & 13,4 & \\
2 &
\end{tabular}

* Valores expressos pela Média \pm DP, demais dados expressos em porcentagem

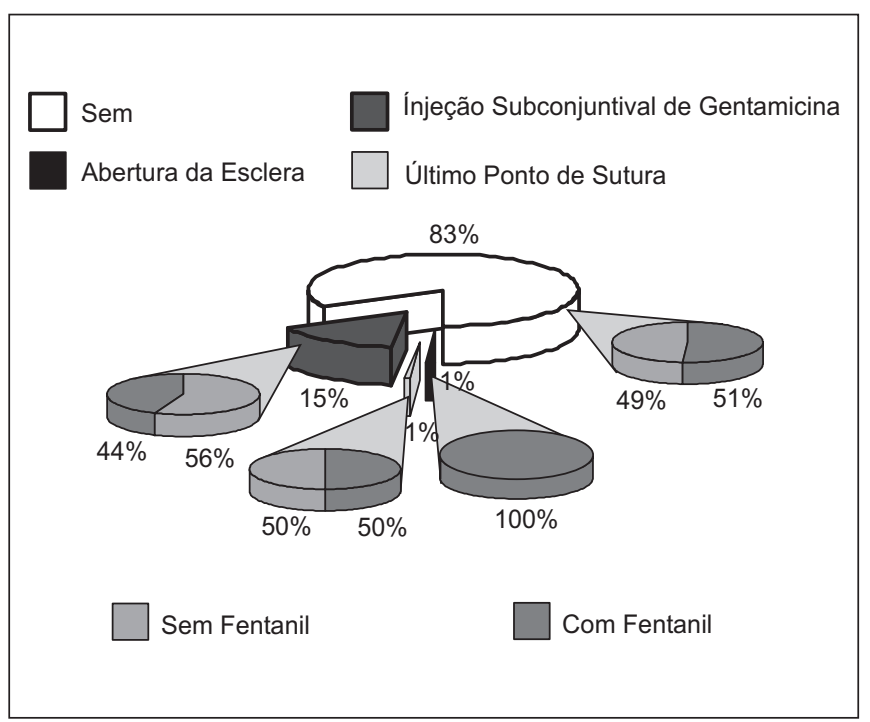

Figura 1 - Distribuição Percentual dos Pacientes em Relação à Dor Per-Operatória, o Momento de Surgimento desta e o Uso ou Não do Fentanil

Após o primeiro bloqueio extraconal não se observou diferença estatística entre os grupos quanto à movimentação das pálpebras e dos músculos reto superior, reto inferior, reto lateral, oblíquo superior, oblíquo inferior e do reflexo de Bell. Os grupos onde não foi utilizado o fentanil apresentaram índice de falhas maior no bloqueio do músculo reto medial (Tabela II). 
Tabela II - Distribuição dos Pacientes em Relação à Persistência de Movimentação da Pálpebra, do Bulbo Ocular (considerando-se cada músculo próprio do bulbo isoladamente) e Persistência do Reflexo de Bell após o Primeiro e Segundo Bloqueio Intracavitário

\begin{tabular}{ccccccccc}
\hline & Palp & RS & RI & RL & RM & OS & OI & RB \\
\hline $1^{\circ}$ Bloqueio & & & & & & & & \\
SF $(n=82)$ & 52,4 & 19,5 & 22,0 & 26,8 & $32,9 *$ & 13,4 & 18,3 & 13,4 \\
CF $(n=82)$ & 54,9 & 20,7 & 22,0 & 22,0 & 17,1 & 7,3 & 8,5 & 3,7 \\
$2^{\circ}$ Bloqueio & & & & & & & & \\
SF $(n=39)$ & 0,0 & 5,1 & 10,3 & 12,8 & 12,8 & 2,6 & 2,6 & 5,1 \\
CF $(n=35)$ & 0,0 & 0,0 & 2,9 & 2,9 & 0,0 & 0,0 & 0,0 & 0,0 \\
\hline
\end{tabular}

Dados apresentados pela porcentagem da persistência de movimentação dos músculos; onde: Palp = movimentação das pálpebras, RS = movimentação do músculo reto superior, $\mathrm{RI}=$ movimentação do músculo reto inferior, $\mathrm{RL}=$ movimentação do músculo reto lateral, $\mathrm{RM}=$ movimentação do músculo reto medial, OS = movimentação do músculo oblíquo superior, $\mathrm{OI}$ = movimentação do músculo oblíquo inferior, RB = reflexo de Bell

${ }^{*} p<0,05=$ estatisticamente significativo

Não se observou diferença estatisticamente significante em relação ao número de bloqueios levando-se em consideração todos os bloqueios realizados (Figura 2).

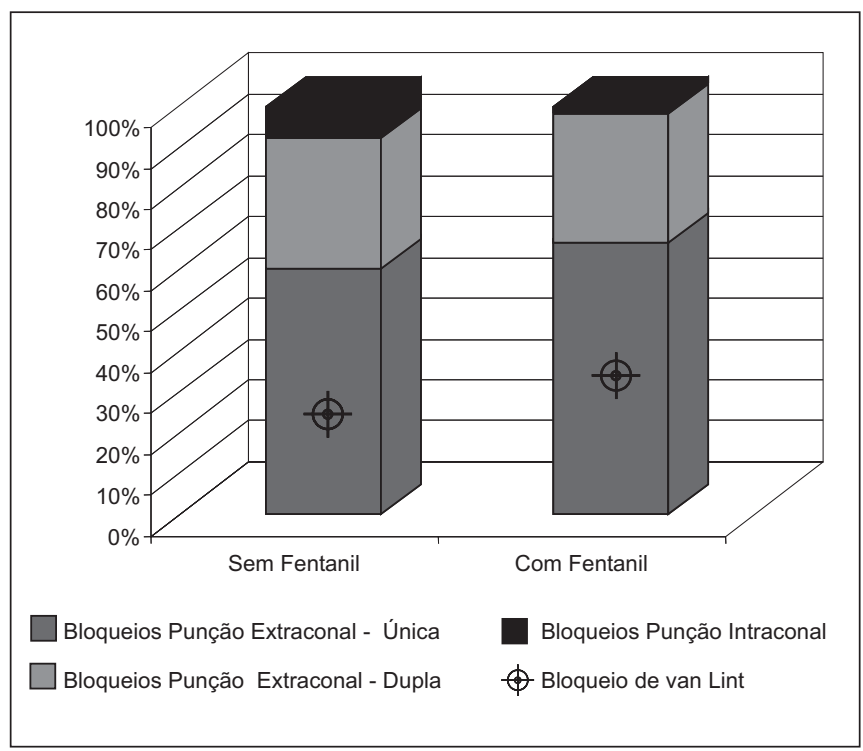

Figura 2 - Distribuição Percentual dos Bloqueios em Relação às Punções

Não há indícios de diferença estatística entre os dois grupos quanto à avaliação do bloqueio pelos cirurgiões, havendo um predomínio de avaliação 1 (anestesia completa, paralisia ocular total, paciente colaborativo), seguida da 2 (anestesia completa, paralisia ocular incompleta, paciente colaborativo) (Tabela III).
Tabela III - Distribuição quanto à Avaliação do Bloqueio pelo Cirurgião

\begin{tabular}{lcccc}
\hline Qualidade & $1(\%)$ & $2(\%)$ & $3(\%)$ & $4(\%)$ \\
\hline SF $(n=82)$ & 90,2 & 8,5 & 1,2 & 0,0 \\
CF $(n=82)$ & 92,7 & 4,9 & 2,4 & 0,0 \\
\hline
\end{tabular}

1 = anestesia completa, paralisia ocular total, paciente colaborativo; 2 = anestesia completa, paralisia ocular incompleta, paciente colaborativo; $3=$ anestesia incompleta, paralisia ocular total, paciente com desconforto cirúrgico e 4 = anestesia incompleta, paralisia ocular incompleta, paciente com desconforto

O surgimento da dor nas primeiras 72 horas de pós-operatório com necessidade de consumo de analgésicos não opióides foi maior nos grupos em que não se utilizou o fentanil (Tabela IV).

Tabela IV - Distribuição dos Pacientes quanto ao Surgimento de Dor (necessidade de consumo de analgésicos não opióides) e o Tempo (em minutos) no Período Pós-Operatório

\begin{tabular}{lcc}
\hline & $\begin{array}{c}\text { SF } \\
(n=82)\end{array}$ & $\begin{array}{c}\text { CF } \\
(n=82)\end{array}$ \\
\hline Dor (\%) & $41,5^{*}$ & 20,7 \\
Tempo (Média \pm DP) & $832,1 \pm 1589,5$ & $1042 \pm 962,4$ \\
\hline
\end{tabular}

* $p<0,05=$ estatisticamente significativo

\section{DISCUSSÃO}

Os dados antropométricos, assim como o olho operado estão distribuídos homogeneamente entre os grupos com isso pode-se garantir que possíveis diferenças nos resultados não sejam decorrentes destes.

Como a doença ocular em questão, não põe a vida do paciente em risco, assim como não se trata de urgência ou emergência, normalmente há tempo hábil para compensar as doenças associadas, justificando assim as boas condições em que estes pacientes freqüentemente se encontram na sala cirúrgica.

Não foi encontrada diferença estatisticamente significante entre os grupos estudados quanto à dor per-operatória. $A$ queixa de dor ocorreu com maior freqüência no momento da injeção subconjuntival da gentamicina, ressaltando a importância desse momento cirúrgico. Não foram encontrados relatos na literatura sobre tal fato.

No presente estudo, a incidência de falhas na acinesia das pálpebras não apresentou diferença estatística entre os grupos. Na avaliação da movimentação do bulbo ocular, considerando-se cada um dos músculos isoladamente, não se observou entre os grupos diferença estatisticamente significante após o primeiro bloqueio intracavitário nos músculos reto superior, reto inferior, reto lateral, oblíquo superior e oblíquo inferior.

O músculo reto medial apresentou maior incidência de falha da acinesia nos bloqueios em que não se utilizou fentanil, não sendo encontrado na literatura explicações para tal fato. 
A avaliação da técnica anestésica e conforto cirúrgico pelo cirurgião não apresentou diferença estatisticamente significava entre os grupos, nos quais $91,5 \%$ receberam nota I (anestesia completa, paralisia ocular total, paciente colaborativo). Campos e col. ${ }^{3}$ relataram que $98 \%$ dos bloqueios foram classificados como ótimos pelos cirurgiões.

Ahmad e col. ${ }^{4}$ relataram a necessidade de rebloqueios intracavitários de $3 \%$ por falha na acinesia do olho, valendo lembrar que estes autores realizam técnica por duas punções, uma superior e outra inferior. Nessa situação, em nosso trabalho encontramos $4,3 \%$ de pacientes que necessitaram do terceiro bloqueio.

Os mesmos autores relataram que nesses bloqueios o músculo reto medial é o que apresenta maior número de falhas de acinesia. No presente estudo foi encontrado maior índice de falhas do músculo reto lateral, seguido da falha do reto medial e inferior.

Katayama e col. ${ }^{5}$ citam que a técnica peribulbar com a injeção em apenas um local por meio de agulha de pequeno calibre apresenta alta incidência de falhas, optando, por esse motivo, pelo bloqueio na dupla punção.

O estudo da analgesia é muito complexo porque expressa o caráter subjetivo das respostas. Há consenso sobre a individualidade das pessoas, ou seja, cada um de nós pode responder diferentemente diante do mesmo estímulo, na dependência do limiar de cada um. O conceito de limiar refere-se ao ponto em que se começa a sentir dor, enquanto tolerância seria a intensidade de dor que se é capaz de sentir antes de fugir do estímulo ${ }^{6}$. Estes fatos revelam importante aspecto concernente à sensação da dor, isto é, existe variabilidade.

Coderre ${ }^{7}$ ressalta que as vias dolorosas são um sistema dinâmico ativo, que tanto responde às sensações dolorosas de maneira complexa como contribui com elas. Alesão tecidual e a dor aguda produzem efeitos intensos no sistema nervoso periférico e central, que alteram a sensibilidade a estímulos subseqüentes. Embora a dor seja útil para alertar ao indivíduo a presença de lesão tecidual, surge o estado de dor patológica quando por algum motivo a dor aguda torna-se persistente.

Portanto, se houver persistência da dor aguda, pode ter inicio o processo de dor patológica. Sendo assim, o tratamento da dor aguda é imperativo, sendo fundamental um método de analgesia pós-operatória eficiente.

Um dos métodos empregados na atualidade de analgesia pós-operatória com eficácia comprovada é a infusão de opióide associado ao anestésico local. Formulamos a hipótese do uso de opióides associado à solução anestésica para a realização de bloqueio oftalmológico, na expectativa de melhorar a analgesia pós-operatória nesses pacientes.

Não foram encontradas referencias da existência de receptores opióides no cone, mas os opióides podem ter ação antálgica por atuarem em receptores opióides das fibras que transmitem impulsos dolorosos (fibras terminais C).

No presente estudo, utilizou-se como método de avaliação da analgesia pós-operatória a necessidade de complemen- tação analgésica por meio da dipirona ou do ácido acetil salicílico.

A dor nas primeiras 72 horas do pós-operatório apresentou maior incidência no grupo sem fentanil, resultado este, estatisticamente significante. Tal fato poderia ser explicado pela ação dos opióides nas fibras $C$ produzindo analgesia ou devido a sua alta redistribuição plasmática e sistêmica, sendo rapidamente redistribuído para o cérebro, sem ação local. Estudou-se também o tempo que decorreu entre a injeção da solução anestésica local e aquele em que os pacientes com dor ingeriram o analgésico no período pós-operatório. Não foram encontrados resultados estatisticamente significantes; tal fato pode ser decorrente da grande variabilidade em relação ao tempo entre os grupos.

Nas condições deste estudo o fentanil melhorou a qualidade do bloqueio quanto a motilidade do músculo reto medial e diminuiu a necessidade de analgésicos no pós-operatório.

\section{Extraconal Block for Cataract Extraction Surgery with Implantation of Intraocular Lens: Contribution of Fentanyl Associated to Local Anesthetics for Quality of Block and Postoperative Analgesia}

Daniel Espada Lahoz, M.D.; Eloisa Bonetti Espada, M.D.; José Carlos Almeida Carvalho, TSA, M.D.

\section{INTRODUCTION}

When submitted to any surgical procedure, although preferring intraoperative anesthesia, patients very often end up tolerating and considering normal the discomfort of postoperative pain ${ }^{1}$. Anesthesiologists have recently given preference to regional anesthetic techniques. This increasing interest is probably due to techniques simplicity, better understanding of their action mechanisms and even the development of new drugs used both in anesthesia and postoperative analgesia, which have improved anesthetic-surgical procedure safety and patients' comfort in the postoperative period ${ }^{2}$.

Although there are no evidences of opioid receptors in the eye ${ }^{3,4}$, it is not known whether opioids for eye surgery blockade would act as in other regional techniques. This study aimed at evaluating the effect of fentanyl on extraconal eye block and postoperative analgesia.

\section{METHODS}

After the approval of the Research Ethics Committees of Hospitals involved, participated in this study 164 patients of both genders, aged 23 to 92 years, physical status ASA I to IV, who were randomly distributed in two groups: Group WF: extraconal block with $0.75 \%$ bupivacaine associated to fentanyl; Group WOF: 0.75\% bupivacaine without fentanyl. 
All patients had surgical indication for cataract extraction and implantation of intraocular lens and blockades were induced by the same anesthesiologist. Anesthetic solution was prepared by a nurse trained for such, who would randomly prepare the solution and give it to the anesthesiologist labeled with a number which would later be compared to other patient's data to define the group patient belonged to.

All patients were monitored with cardioscopy, non-invasive blood pressure and pulse oximetry before peripheral venoclysis in an upper limb with 20 or $22 \mathrm{G}$ catheter. Hydration was then started with lactated Ringer's solution at the approximate speed of $3 \mathrm{ml} \cdot \mathrm{min}^{-1}$.

Anesthetic solution was $0.75 \%$ bupivacaine with hyaluronidase (100 UTR. $\mathrm{min}^{-1}$ ) in a total volume of $7 \mathrm{ml}$. Hyaluronidase was diluted in bupivacaine at a ratio of 2,000 UTR for each $20 \mathrm{ml}$ of $0.75 \%$ bupivacaine. The fentanyl group also received $0.5 \mathrm{ml}$ fentanyl $(25 \mu \mathrm{g})$. The group without fentanyl received $0.5 \mathrm{ml}$ distilled water. Blockade was induced with disposable $7 \mathrm{G}$ needle with $40 \mathrm{~mm}$ length.

Patients were sedated with $50 \mu \mathrm{g}$ intravenous fentanyl and titrated doses of $0.5 \%$ propofol until loss of ciliary reflex, when extraconal block was induced. When emerging from sedation, patient was evaluated in terms of akinesia and analgesia.

Upper extraconal access had as reference 1 to $2 \mathrm{~mm}$ from the lateral border of the supra-orbital rim, directing the needle to the orbit until it crossed skin and subcutaneous tissue. Needle point was redirected to the upper orbital fissure, approximating it as close as possible to bone structures. After approximation, which occurred at 30 to $35 \mathrm{~mm}$, needle was retracted for approximately 1 to $2 \mathrm{~mm}$ where anesthetic solution was injected.

Lower extraconal access had as puncture site the junction of the lateral third with the medial third of the lower orbital rim. Needle point was directed to the lower orbital fissure being introduced in parallel to the orbital floor for approximately 30 $\mathrm{mm}$, where anesthetic solution was injected.

Additional blockade was induced in case of proposed blockade failure. If upper extraconal block failed, it was complemented by lower extraconal block, and vice versa. Anesthetic solution was $0.75 \%$ bupivacaine with hyaluronidase (100 UTR. $\mathrm{ml}^{-1}$ ), in a total volume of $3 \mathrm{ml}$. If additional block failed, a third intraconal or retrobulbar blockade was induced with $0.75 \%$ bupivacaine and hyaluronidase (100 UTR. $\mathrm{ml}^{-1}$ ), in a total volume of $3 \mathrm{ml}$.

Intraconal block access was the junction of the lateral third with the medial third of the lower orbital rim. When the needle point surpassed eye Ecuador, it was supero-medially repositioned toward the junction of the medial and lateral thirds of the upper orbital rim of the contralateral eye. This procedure persisted until a sensation of perforating a paper sheet and anesthesia was administered at this point.

When there was only motor eyelid fail, anesthesia was complemented with modified van Lint blockade using $3 \mathrm{ml}$ of $0.75 \%$ bupivacaine alone.

Modified van Lint blockade was induced by subcutaneously injecting anesthetic solution approximately $1 \mathrm{~cm}$ from the lateral orbital rim, directly over the periostium, directing the needle caudally and then cephalically and injecting $1.5 \mathrm{ml}$ in each direction.

After local anesthetic injection, firm digital pressure was exerted on the eye, in any stage, for approximately 2 to 3 minutes, after which a $650 \mathrm{~g}$ weight was placed on the eye to be operated, aiming at decreasing intraocular pressure and helping local anesthetic spread.

The following parameters were studied to evaluate blockade quality: intraoperative pain, eyelid motility maintenance, eyeball movement (considering extra-ocular muscles in association and separately), Bell's reflex persistence, number of additional extracavitary blocks needed to obtain surgical conditions and block evaluation by surgeons, who were blind to the injected solution and blockade access way, according to the following scale:

1) Complete anesthesia, total eye paralysis, cooperative patient;

2) Complete anesthesia, incomplete eye paralysis, cooperative patient;

3) Incomplete anesthesia, total eye paralysis, patient with surgical discomfort;

4) Incomplete anesthesia, incomplete eye paralysis, patient with discomfort.

Anesthesia during the first 72 postoperative hours was evaluated by patients' request for analgesia, which consisted of oral dipirone or AAS. Time elapsed from blockade until drug administration was also evaluated in those patients.

Chi-square test was used for statistical analysis to check differences in the distribution of one categorized attribute as a function of another categorized parameter. In some cases the test could not be applied due to the low frequency of some attributes. When cases were more than 40 , Chi-square test with Yates correction was used, as well as for cases between 20 and 40, and no attribute had a frequency lower than 5. When cases were lower than 20 and attribute frequency lower than 5, Fisher's test was used.

Analysis of Variance (ANOVA) was used to check differences in age, weight, height and time in minutes from blockade to first postoperative pain complaint.

Significance level was $5 \%$ for all statistical tests and tests were performed with the aid of the statistical software SPSS for Windows, release 6.0.

\section{RESULTS}

Patients were of both genders (78 males and 86 females), aged less than 23 years to more than 92 years, with homogenous distribution between groups in demographics and operated eye (Table I).

There were no statistically significant differences between groups in physical status and Goldman's cardiac risk index, with prevalence of physical status ASA Il and Goldman's cardiac risk index 1 (Table I).

Revista Brasileira de Anestesiologia Vol. 53, N 5, Setembro - Outubro, 2003 
Table I - Demographics Data, Operated Eye, Physical Status and Goldman's Cardiac Risk Index

\begin{tabular}{lcc}
\hline Variables & $\begin{array}{c}\text { Group WF } \\
(\mathrm{n}=82)\end{array}$ & $\begin{array}{c}\text { Group WOF } \\
(\mathrm{n}=82)\end{array}$ \\
\hline Age (years) & $69.7 \pm 10.3$ & $68.4 \pm 12.8$ \\
Weight $(\mathrm{kg})^{*}$ & $67.4 \pm 16.2$ & $65.9 \pm 12.6$ \\
Height (cm)* & $164.2 \pm 11.3$ & $161.7 \pm 10.7$ \\
Gender (\%) & & \\
$\quad$ Male & 50 & 54.9 \\
$\quad$ Female & 50 & 45.1 \\
Operated Eye (\%) & & \\
Right & 54.9 & 46.3 \\
$\quad$ Left & 45.1 & 53.7 \\
Physical Status (\%) & & \\
ASA I & 18.3 & 32.9 \\
ASA II & 73.2 & 56.1 \\
ASA III & 6.1 & 11 \\
ASA IV & 2.4 & 0 \\
Goldman (\%) & & 89 \\
1 & 86.6 & 11 \\
2 & 13.4 &
\end{tabular}

*Values expressed in Mean $\pm \mathrm{SD}$, remaining data expressed in percentages

There were no statistical differences in intraoperative pain between groups in the different moments (sclera opening, last scleral suture and subconjunctival gentamicine injection). There has been a low incidence of perioperative pain in all moments, with a mild increase at subconjunctival gentamicine injection, as shown in figure 1.

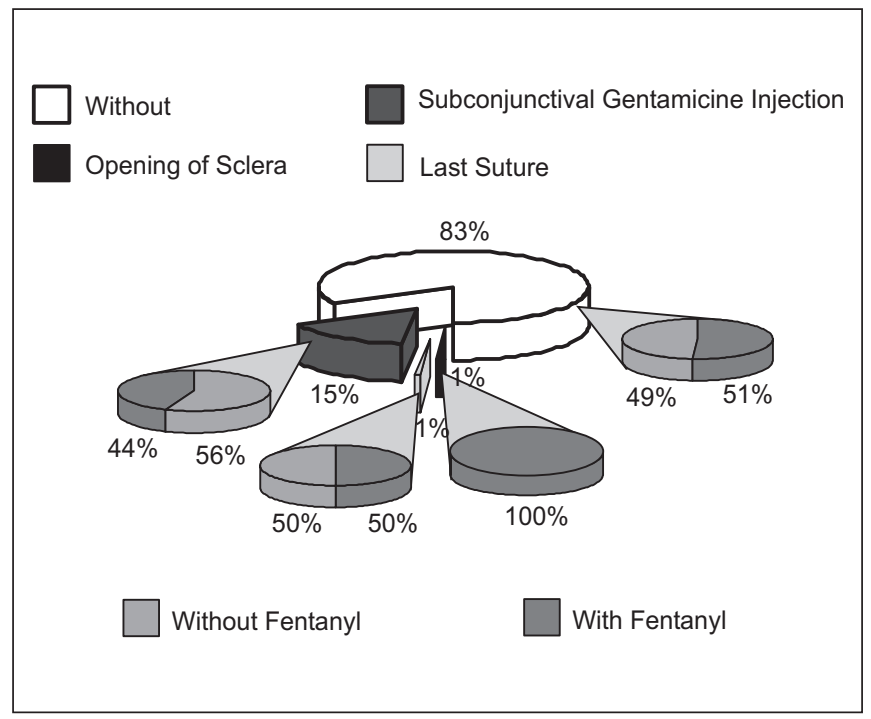

Figure 1 - Patients Distribution (in \%) as to Intraoperative Pain, Beginning of Pain and Use or Not of Fentanyl

Revista Brasileira de Anestesiologia

Vol. 53, № 5 , Setembro - Outubro, 2003
There were no statistical differences between groups in eyelid and upper rectus, lower rectus, upper oblique muscles and Bell's reflex after first extraconal block. Group not receiving fentanyl had a higher medial rectus muscle fail rate (Table II).

Table II - Distribution of Patients in Relation to Eyelid, Eyeball Movement (considering each muscle separately) and Bell's Reflex Persistence after First and Second Intracavitary Block

\begin{tabular}{ccccccccc}
\hline & Eyelid & SR & IR & LR & MR & SO & IO & BR \\
\hline $1^{\text {st }}$ Blockade & & & & & & & & \\
WF $(n=82)$ & 52.4 & 19.5 & 22.0 & 26.8 & $32.9 *$ & 13.4 & 18.3 & 13.4 \\
WOF $(n=82)$ & 54.9 & 20.7 & 22.0 & 22.0 & 17.1 & 7.3 & 8.5 & 3.7 \\
$2^{\text {nd }}$ Blockade & & & & & & & & \\
WF $(n=39)$ & 0.0 & 5.1 & 10.3 & 12.8 & 12.8 & 2.6 & 2.6 & 5.1 \\
WOF $(n=35)$ & 0.0 & 0.0 & 2.9 & 2.9 & 0.0 & 0.0 & 0.0 & 0.0 \\
\hline
\end{tabular}

Data in percentage of muscles movement persistence; where: Eyelid = eyelid movement, $\mathrm{SR}=$ superior rectus muscle movement, $\mathrm{IR}=$ inferior rectus muscle movement, LR - lateral rectus muscle movement, $\mathrm{MR}=$ medial rectus muscle movement, $\mathrm{SO}=$ superior oblique muscle movement, $\mathrm{IO}=$ inferior oblique muscle movement, BR = Bell's reflex

${ }^{*} p<0.05=$ statistically significant

There were no statistically significant differences in the number of blockades, taking into consideration all blockades induced (Figure 2).

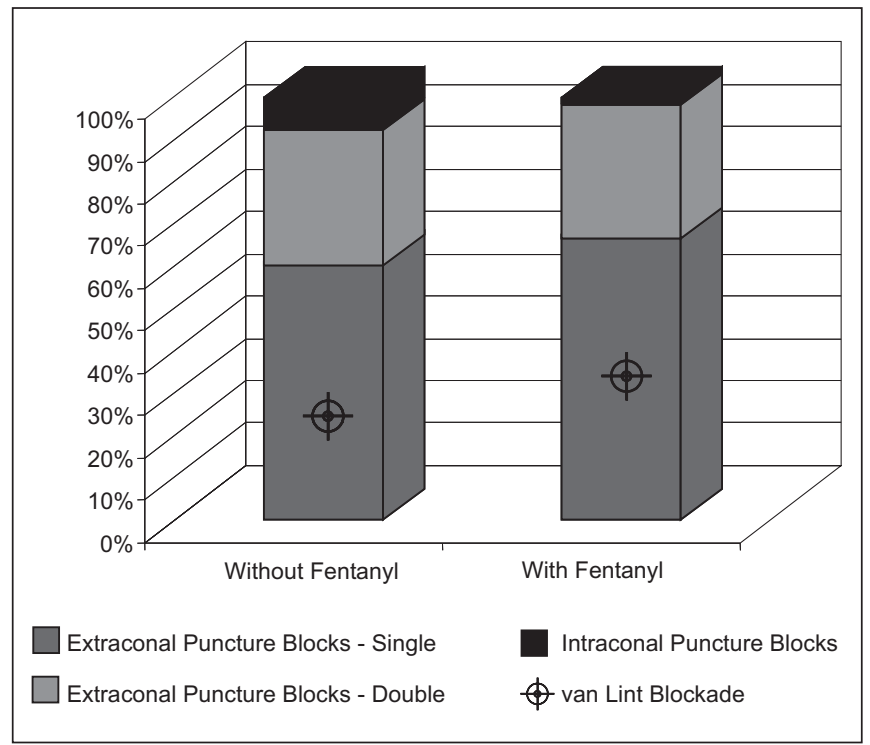

Figure 2 - Blockades Distribution (\%) with Relation to Punctures

There were no statistically significant differences between groups in blockade evaluation by surgeons, with a predominance of evaluation 1 (complete anesthesia, total eye paralysis, cooperative patient), followed by 2 (complete anesthesia, incomplete eye paralysis, cooperative patient) (Table III). 
Table III - Blockade Evaluation by Surgeon

\begin{tabular}{lcccc}
\hline Quality & $1(\%)$ & $2(\%)$ & $3(\%)$ & $4(\%)$ \\
\hline WF $(n=82)$ & 90.2 & 8.5 & 1.2 & 0.0 \\
WOF $(n=82)$ & 92.7 & 4.9 & 2.4 & 0.0 \\
\hline
\end{tabular}

$1=$ total anesthesia, total eye paralysis, cooperative patient; 2 = total anesthesia, incomplete eye paralysis, cooperative patient; $3=$ incomplete anesthesia, total eye paralysis, patient with surgical discomfort; and $4=$ incomplete anesthesia, incomplete eye paralysis, patient with discomfort

Pain in the first 72 postoperative hours requiring non-opioid analgesics was higher in the group without fentanyl (Table IV).

Table IV - Distribution of Patients as to Beginning of Pain (need for non-opioid analgesics) and Time (in minutes) in the Postoperative Period

\begin{tabular}{lcc}
\hline & $\begin{array}{c}\text { WF } \\
(\mathrm{n}=82)\end{array}$ & $\begin{array}{c}\text { WOF } \\
(\mathrm{n}=82)\end{array}$ \\
\hline Pain (\%) & $41.5^{*}$ & 20.7 \\
Time (Mean \pm SD) & $832.1 \pm 1589.5$ & $1042 \pm 962.4$ \\
\hline
\end{tabular}

${ }^{*} p<0.05=$ statistically significant

\section{DISCUSSION}

Demographics data and operated eye were homogeneous between groups so possible differences in results are not a consequence of such data.

Since this eye disease is neither life threatening nor an urgency or emergency, in general there is enough time to compensate associated diseases, thus justifying the good conditions presented by patients in the operating room.

There were no statistically significant differences between groups in intraoperative pain. Pain complaint was more frequent at subconjunctival gentamicine injection, stressing the importance of this surgical step. No reports in the literature were found on this fact.

In our study, the incidence of eyelid akinesia failure was not statistically different between groups. In evaluating eyeball movement and considering each muscle separately, there were no statistically significant differences between groups after the first intracavitary block of superior rectus, inferior rectus, lateral rectus, superior oblique and inferior oblique muscles.

Akinesia failure was higher in the medial rectus muscle in blockades without fentanyl and no explanation was found in the literature for such fact.

Technical anesthetic evaluation and surgeons' evaluation of surgery were not statistically different between groups: $91.5 \%$ received grade 1 (complete anesthesia, total eye paralysis, cooperative patient). Campos et al. ${ }^{3}$ have reported $98 \%$ of blockades considered very good by surgeons.

Ahmad et al. ${ }^{4}$ have reported $3 \%$ intracavitary re-blockades due to eye akinesia failure, but it is worth mentioning that these authors perform the technique with two punctures (upper and lower). In our study we have found $4.3 \%$ of patients needing a third block.
The same authors have reported that in such blockades, medial rectus muscle presents the highest incidence of akinesia failure. We have found the highest incidence of failures in the lateral rectus muscle, followed by medial and inferior rectus muscle.

Katayama et al. ${ }^{5}$ mention that the peribulbar technique with single injection and fine needle has a high incidence of failures and for this reason they have adopted the double puncture block.

The study of analgesia is very complex because responses are subjective. There is a consensus on the individuality of people, that is, each one of us may respond differently to the same stimulation, depending on individual thresholds. Threshold is the moment in which one starts to fell pain, while tolerance would be the intensity of pain one is able to stand before running away from stimulation ${ }^{6}$. These facts reveal an important aspect related to pain sensation, that is, there is variability.

Coderre ${ }^{7}$ highlights that pain pathways are an active dynamic system both responding to pain sensations in a complex manner and contributing to them. Tissue injury and acute pain produce intensive effects on peripheral and central nervous system, which change the sensitivity to subsequent stimulations. Although pain is useful to alert about tissue injury, there is the state of pathological pain when for some reason acute pain becomes persistent.

So, if there is acute pain persistence, a pathological pain process may follow. In this case, acute pain treatment is mandatory, and an efficient postoperative analgesia method is critical.

One proven method currently used in postoperative analgesia is the infusion of opioids associated to local anesthetics. We have developed the hypothesis of using opioids associated to anesthetic solution for eye blockade in an attempt to improve postoperative analgesia.

There are no references of opioid receptors in the cone, but opioids may have analgesic effects for acting on opioid receptors of fibers transmitting painful impulses (terminal $C$ fibers). Postoperative analgesia was evaluated in our study by the need for analgesic complementation with dipirone or acety salicilic acid (AAS).

The incidence of pain during the first 72 postoperative hours was higher in the group without fentanyl being this result statistically significant. Such fact could be explained by the action of opioids on $\mathrm{C}$ fibers producing analgesia, or by to their high plasma and systemic redistribution, being promptly redistributed to brain without local action.

We have also studied time elapsed between local anesthetic injection and the moment in which patients with pain have requested analgesics in the postoperative period. Results were not statistically significant, which may have been due to major variability of time between groups.

In the conditions of our study, fentanyl has improved blockade quality in terms of medial rectus muscle motility and has decreased the need for postoperative analgesics. 


\section{REFERÊNCIAS - REFERENCES}

01. Eisenach JC - Incidência da Dor Pós-Operatória e Fatores Predisponentes. em: Bonnet F - A Dor no Meio Cirúrgico, Porto Alegre, Artes Médicas Sul, 1993;48-56.

02. Rocha MEAFPG - Efeito da Temperatura do Anestésico Local na Dispersão Subaracnóide da Bupivacaína Hiperbárica a 0,5\%. Dissertação (Mestrado), Faculdade de Medicina, Universidade de São Paulo, 1997:147.

03. Campos AR, Azevedo FJT, Silva LC - Bloqueio peribulbar: uma alternativa para cirurgia oftálmica. Rev Bras Anestesiol, 1989;39:287-291.

04. Ahmad S, Ahmad A, Benzon HT - Clinical experience with the peribulbar block for ophthalmologic surgery. Reg Anesth, 1993;18:184-188.

05. Katayama M, Zambotti HC, Vieira JL et al - Bloqueio peribulbar e retrobulbar em cirurgia oftálmica. Estudo clínico comparativo com bupivacaína a 0,75\%, com e sem hialuronidase. Rev Bras Anestesiol, 1993:43:159-166.

06. Loeser JD, Butler SH, Chapman CR et al - Bonica's Management of Pain, $3^{\text {rd }}$ Ed, Philadelphia, Lippincott Williams $\varepsilon$ Wilkins, $2001 ; 1-264$.

07. Coderre TJ - Conseqüências fisiológicas da lesão tecidual e da dor aguda. Clin Anest Am Norte. 1992;10:243-265.

\section{RESUMEN}

Lahoz DE, Espada EB, Carvalho JCA-Bloqueo Extraconal para Facectomia con Implantación de Lente Intra-Ocular: Influencia del Fentanil Asociado al Anestésico Local en la Calidad del Bloqueo y en la Analgesia Pos-Operatoria

JUSTIFICATIVA Y OBJETIVOS: La anestesia locorregional para cirugías oftalmológicas ofrece ventajas, como: mínimas alteraciones fisiológicas, anestesia completa, bloqueo de los reflejos oculares, pequeña incidencia de náuseas y vómitos, menor tiempo de recuperación y analgesia pos-operatoria. La preocupación constante con la calidad del bloqueo, así como la abordaje de la analgesia pos-operatoria debe quedar bajo la responsabilidad del anestesiologista. El objetivo de este estudio fue evaluar si el fentanil contribuye en la calidad del bloqueo extraconal y en la analgesia pos-operatoria de facectomias con implantación de lente intra-ocular.

MÉTODO: Se estudió la asociación del fentanil y bupivacaína a $0,75 \%$ en la calidad del bloqueo ocular y en la analgesia pos-operatoria en 164 pacientes sometidos a facectomia con implantación de lente intra-ocular (técnica extracapsular), de ambos sexos con homogeneidad de parámetros antropométricos, ojo operado, clasificación del estado físico (ASA) e índice de riesgo cardíaco de Goldman. Los pacientes fueron distribuidos en dos grupos (82 pacientes en cada grupo) por sorteo de forma aleatoria, con y sin fentanil. Se evaluó la calidad del bloqueo por: aparecimiento de dolor en el per-operatorio, manutención de movimentación de los párpados o del globo ocular, persistencia del reflejo de Bell, número de bloqueos realizados para la obtención de condiciones quirúrgicas y evaluación del bloqueo por el cirujano. La analgesia pos-operatoria fue evaluada por la necesidad de complementación analgésica por el paciente.

RESULTADOS: Fentanil asociado a la solución anestésica en el bloqueo extraconal aumentó significativamente el bloqueo del músculo recto medial (con fentanil - 17,1\%, sin fentanil$32,9 \%)$ y diminuyó el consumo de analgésicos en el período pos-operatorio (uso de analgésicos con fentanil - 20,7\%, en el uso de analgésicos con fentanil - 41,5\%).

CONCLUSIONES: En las condiciones de este estudio el fentanil mejoró la calidad del bloqueo cuanto a la motilidad del músculo recto medial y diminuyó la necesidad de analgésicos en el pos-operatorio. 